\title{
Underlying Sociocultural Practices Influencing Prevalence of Female Genital Mutilation/Cutting in Kajiado County
}

\author{
Bernard Mbogo*, Sarah Karanja, Kennedy Omwaka, Denge Lugayo, Charles Leshore \\ Amref Health Africa, Nairobi, Kenya \\ Email: *Bernard.mbogo@amref.org
}

How to cite this paper: Mbogo, B., Karanja, S., Omwaka, K., Lugayo, D. and Leshore, C. (2019) Underlying Sociocultural Practices Influencing Prevalence of Female Genital Mutilation/Cutting in Kajiado County. Advances in Sexual Medicine, 9, 17-28.

https://doi.org/10.4236/asm.2019.92002

Received: January 28, 2019

Accepted: April 22, 2019

Published: April 25, 2019

Copyright $\odot 2019$ by author(s) and Scientific Research Publishing Inc. This work is licensed under the Creative Commons Attribution International License (CC BY 4.0).

http://creativecommons.org/licenses/by/4.0/

\begin{abstract}
Background: Female Genital Mutilation/Cutting (FGM/C) often has lifelong negative consequences for a woman's physical and mental health but is still practiced in some parts of Kenya including Kajiado County. We aimed to estimate the current prevalence as well as the socio-cultural beliefs and power relations that are in favor of or against the practice in Kajiado, Kenya. Methods: A mixed method cross-sectional study was conducted in Kajiado County. The study targeted: women of reproductive age (15 to 49 years); community health volunteers (CHVs); opinion leaders; health care workers; officials from the ministries of Education, Health, Culture, Gender and Social Services; Community Health Assistants (CHAs); Traditional Birth Attendants (TBAs); teachers; morans and adolescent boys and girls aged 10 to 24 years. Data were collected both quantitatively through a household questionnaire and qualitatively through the focus group discussions and key informant interviews. Factors influencing Female Genital Mutilation/Cutting (FGM/C) were classified as either social, cultural beliefs or economic. Results: From the study, quantitative results revealed that the prevalence of FGM/C in Kajiado County was $91 \%$, with most of them (96.7\%) practicing type 2 (excision) circumcisions. From the interviews, girls undergo the cut as a rite of passage to womanhood and thus a prerequisite for marriage. It is also believed that girls who are uncircumcised cannot be helped by TBAs in delivery. It is believed that their blood is poisonous and can cause bad omen to whoever comes in contact with dirty blood. Additionally, it is believed that girls go through the cut to avoid conflict and natural phenomena; for instance, drought and outbreaks of diseases that kill many people. Finally, it is a practice that earns respect for the parents of the girls and incentives as dowry to the father of the girl. TBAs that perform FGM/C get paid in cash and kind. Conclusion: Female genital mutilation/cutting practice in Kajiado County is still high. Ef-
\end{abstract}


forts to end the practice will need to have an integrated approach to include all the players. Suggested alternatives to the cut must, therefore, be inclusive so as to address the myths/beliefs, misconceptions, socio-cultural and economic factors in favor of the vice. The alternatives must be inclusive for the beneficiaries, supporters, and practitioners.

\section{Keywords}

Female Genital Mutilation, Alternative Rite of Passage, Prevalence, Kajiado County, Kenya

\section{Introduction}

The International Human Rights law including the Universal Declaration of Human Rights (1948) proclaims the right for all human beings to live in conditions that enable them to enjoy good health and access health care. Female genital mutilation or female genital cutting (FGM/C) is the ritual practice of cutting or removing of some or all of the external female genitalia. It is usually classified into four major types: Type 1-Clitoridectomy, partial or total removal of the clitoris and/or the prepuce; Type 2-Excision, partial or total removal of the clitoris and the labia minora, with or without excision of the labia majora; Type 3-Infibulation, narrowing of the vaginal opening through the creation of a covering seal; and Type 4-All other harmful procedures to the female genitalia for non-medical purposes.

Female Genital Mutilation/Cutting (FGM/C) often has brutal consequences for a woman's physical and mental health [1]. Female Genital Mutilation/Cutting (FGM/C) results in serious health problems such as urinary tract infections, infertility, childbirth complications, abscesses, small benign tumors, hemorrhages, and clitoral cysts. Additionally, depending on the type of FGM/C performed, the immediate health consequences of the procedure can include pain and bleeding that can lead to hemorrhage and even death. Long-term consequences can include: irreversible loss of the clitoris and possibly the outer and inner labia, chronic infection, infertility, difficult pregnancy and childbirth, painful sexual intercourse and menstruation [1].

While the exact number of girls and women worldwide who have undergone Female Genital Mutilation/Cutting (FGM/C) remains unknown, at least 200 million girls and women in 30 countries have been subjected to the practice [2]. Female Genital Mutilation/Cutting (FGM/C) is most common in Western, Eastern and North-Western regions in Africa, some countries in Asia and the Middle East. An estimated 3 million girls are thought to undergo the FGM/C every year [3]. In Kenya, the prevalence of FGM/C amongst girls and women aged 15 49 yrs is $21 \%$ compared with $27 \%$ in $2008-09$ and $32 \%$ in 2003 [4]. Kenya has great ethnic and cultural diversity, as reflected in the differing rates of FGM/C across the ethnic groups, the type of FGM/C performed and the underlying rea- 
sons for practicing it. Among the Somalis, who live predominantly in the North Eastern province, the prevalence of FGM/C is $97.7 \%$ [5], the majority (75\%) of these are of the most severe Type III infibulation. The next highest prevalence is among the Kisii (also known as the Abagussi or Gusii) at $96.1 \%$ and the Maasai at $73.2 \%$. The Abagussi and Maasai practice Type I clitoridectomy and Type II excision respectively [3].

Kajiado County, in Kenya, is mainly inhabited by the Maasai community. In Kajiado most women still believe that undergoing FGM/C is the key to success in all aspects of life as they believe that it is only after undergoing through the cut that a girl is considered to be a woman. Those who support the practice also believe that the practice reduces infidelity and prevents HIV and Aids [5]. Girls who undergo Female genital cutting are usually considered mature and are ready to be married off and start childbearing. By going through FGM/C, they drop out of school, if they are in school; they perform poorly and fail to meet their gender equality rights [6].

Amref Health Africa (https://www.amref.org/) [4] and other child protection partners working in Kajiado County have been reactive in an effort to end the practice, to reduce school dropout and early and forced marriages for girls through the Alternative Rite of Passage (ARP) model. The ARP model was developed by Amref Health Africa and entails mentoring and graduating girls into womanhood without necessarily going through FGM/C. The process is aimed at delaying the girls to get married at an early age, delaying the girl in risks of getting pregnant before 18 years and escape the FGM/C (Substituting the "cut": A Look into Alternative Rites of Passage-Youth For Change: a global platform for girls' rights youth activism n.d.) [7]. The model has been implemented in Kajiado since 2010. There has also been extensive advocacy with the county government of Kajiado as well as the national government to legislate on the enforcement of laws against harmful cultural practices including FGM/C and continuous community sensitization on the dangers and disadvantages of the FGM/C. Despite many years of intervention by Amref Health Africa and other child protection agencies, FGM/C prevalence still remains high in the Maasai communities (73.2\%) which Kajiado County is part of even though there are no county-specific estimates for Kajiado.

Despite the full support of the political and legal structures, there might be other socio-cultural push factors that could be overlooked by the current interventions that are maintaining the high prevalence of FGM/C in Maasai communities like Kajiado. Therefore, to adequately tackle the issue of female genital cutting, a critical review of the beneficiaries, the drivers or supporters of the suggested alternatives are required. According to a recent study by Amref Health Africa on establishing reliable evidence on the effectiveness of the complex interventions towards ending FGM/C is challenging [4].

\section{Objectives of the Study}

- To estimate the Prevalence of Female Genital Mutilation/Cutting (FGM/C) 
among women in Kajiado County.

- To determine the socio-cultural beliefs and power relations that are in favor or against the practice.

- To assess the current Knowledge, Attitudes, and Perceptions (KAP) towards Female Genital Mutilation/Cutting (FGM/C).

\section{Methodology}

\subsection{Study Design}

The study used a mixed methods approach, which included a quantitative household cross-sectional survey augmented with a qualitative study conducted through Focus Group Discussions and Key Informant Interviews. The study was conducted in October 2017.

\subsection{Study Population}

The primary study participants were women of reproductive age (15 to 49 years) and adolescent girls in Kajiado County. Secondary participants included community health volunteers ( $\mathrm{CHVs}$ ), opinion leaders, health care workers, officials from the Ministry of Education, Ministry of Health, Ministry of Culture, Gender and Social Services, Community Health Assistants (CHAs), Traditional Birth Attendants (TBAs), Teachers, morans and adolescent boys and girls aged 10 to 24 years.

\subsection{Sample Size Calculation}

The sample size was determined using Fisher's exact test. The assumption was that the sample would be representative, the sampling error would be small and that the results would be generalizable.

$$
n=\frac{z^{2} p q}{e^{2}} \times 2
$$

where $n=$ sample size.

where $Z=$ value for the selected alpha level (1.96) which corresponds to $95 \%$ confidence interval $p=$ population proportion estimated to have a particular characteristic (where there is no reasonable estimate a default of $50 \%$ or 0.5 is used).

$$
\begin{gathered}
q=1-p, p=0.5, q=0.5, e=0.05, \text { deff }=2 \\
n=\frac{1.96^{2} \times 0.5 \times 0.5 \times 2}{0.05^{2}}=768
\end{gathered}
$$

Hence a total of 768 households were sampled for inclusion in the study.

\subsection{Sampling}

A two-stage probability sampling strategy was used to select the target population. In the first stage, Enumeration Areas (EAs) were selected at random from an updated list of EAs provided by the Kenya National Bureau of Statistics 
(KNBS) for all administrative areas in Kajiado Central, Kajiado South, Kajiado West and Kajiado East sub-counties of Kajiado County. Secondly, households were randomly picked from a list of households-generated by the Community Health Volunteers (CHVs) with supervision by community Health Assistants (CHAs) - in each of the selected EAs from the first stage. The number of households sampled in each EA was allocated proportionally to its size. The randomly selected households had to satisfy the set eligibility criteria to participate in the survey; had a woman of reproductive age that was a permanent resident of the household (not a visitor). If the household did not satisfy the criteria, the next eligible household was selected. The entire process was coordinated by the County and Sub-county Community Health Strategy Focal Persons, the study team and the community guides-who showed the research assistant boundaries to villages. Before the interviews were conducted, consent was sought from the respondents. For the children respondents, assent was sought from their parents/guardians.

\subsection{Pre-Testing of Study Tools}

All the tools were developed by the project team and were reviewed and approved by the Ethics and Scientific Review Committee (ESRC). The tools were pre-tested prior to data collection. A site was selected where the research was not to cover and the tool in the ODK platform in the tablets was used in the pretest. After Pre-testing was done, the study tools were revised accordingly based on the experience from the pretest activity.

\subsection{Data Collection and Management}

Quantitative data was collected electronically using a structured household questionnaire tool programmed in the Open Data Kit (ODK). Qualitative data were collected using semi-structured discussion guides. Discussants were purposefully selected to participate in the discussions due to their previous contact with the project interventions. Key Informants were selected from the relevant departments in the county and who was presumed to be knowledgeable in the subject matter based on their roles.

For the qualitative part, a total of 15 key informant interviews were conducted with the following: County Director of the Ministry of Health (1); The County Director Ministry of Education (1); the County Director Ministry of Culture, Gender and Social Services (1); the County Public Health Officer (1); the County Community Health Strategy Focal Person (1); Four (4) Traditional Birth Attendants; Four (4) Teachers; and two (2) Community Health Workers. A total of 16 focus group discussions were also conducted with adolescent girls in school (4 FGDs); adolescent boys in school (4 FGDs); Men (4 FGDs) and Women (4 FGDs).

\subsection{Data Analysis}

Descriptive analysis of the quantitative data was performed using the SPSS soft- 
ware. For qualitative data, a codebook was developed and coding was done thematically whereby events or occurrences that share the same characteristics were assigned the same code. Pre-set codes were identified from the interview guides and research questions and emerging codes were derived from the transcripts. The coded data was refined by collapsing, expanding or revising the coding categories. The relationship between the quotes and codes was then explored to answer the research questions. Nvivo 11 software was used in the qualitative analysis process.

\section{Results}

\subsection{Quantitative Results}

The survey achieved an overall response rate of $95 \%$ with a total of 732 structured questionnaires being administered to women of reproductive age and adolescents aged $10-19$ yrs against a target of 768 respondents. The prevalence of FGM/C was $91 \%$ in Kajiado County. (Figure 1)

The mean age at which women were circumcised was 14 years with a range of 6 to 25 years. (Table 1 )

Type 2 FGM/C, where flesh is removed, was the most common (performed on 97\% of circumcised women) type of circumcision practiced in Kajiado County, $5 \%$ were nicked (Type 1 ) while $3 \%$ had their genital area sown closed (Type 3 ). In some instances, some of the respondents might have had their flesh removed and their genitals sewn closed. Traditional circumcisers, who also double up as Traditional Birth Attendants (TBAs), carried out the majority (98\%) of circumcisions. When asked where the circumcision was performed, $93 \%$ responded

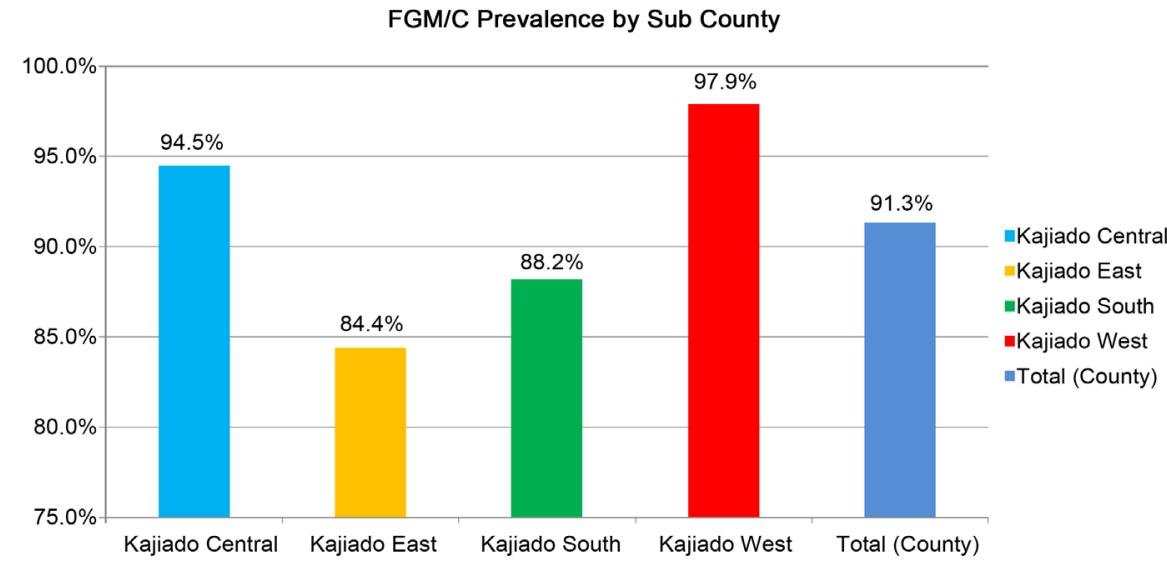

Figure 1. FGM/C prevalence by sub-county.

Table 1. Mean age at which the women were circumcised.

\begin{tabular}{ccccc}
\hline Age & Kajiado Central & Kajiado East & Kajiado South & Kajiado West \\
\hline Mean & 14 & 14 & 15 & 14 \\
Min & 9 & 6 & 8 & 10 \\
Max & 18 & 25 & 21 & 21 \\
\hline
\end{tabular}


that circumcision took place within their homesteads, $4 \%$ of the women were circumcised at a secluded place far from their homes, $1 \%$ at health facility and $2 \%$ across the border in Tanzania where the law against FGM/C exists but is not prohibitively enforced.

The fraction of respondents aware of anti-FGM/C messages, from self-reports, was $95 \%$ (Figure 2). The survey revealed that the sources where the respondents had heard of Anti-FGC messages were Radio 49\%, GOK officials 40\%, Community leaders $32 \%$, Amref staffs $31 \%$, Schools $28 \%$, religious leaders/health workers $23 \%$ while the rest heard from other sources such as peer educators, relatives, and posters. Knowledge levels on alternatives to the cut, about half (48\%) of the respondents were aware of the Alternative Rites of Passage (ARP) as an anti-FGM/C strategy. Among those who reported being aware of ARP, $84 \%$ had a view that FGM/C should be stopped and felt that ARP is acceptable by the community as a means of graduating girls to womanhood. Among those who had not undergone FGM/C, the decision not to undergo FGM/C was made by the women themselves $(54 \%)$, fathers $(22 \%)$ or mothers $(18 \%)$ while others categories of decision makers accounted for $6 \%$.

\subsection{Qualitative Results}

We classified the factors in favor of the continuation of female genital mutilation/cutting into social beliefs, cultural belief, and economic factors. Hindering factors were found to be the government laws, buy in from political leaders and legal structures in place which prohibits the practice. Another factor against the practice is the Alternative rite of passage model by Amref Health Africa which seeks to graduate girls to womanhood without going through the cut.

\subsection{Social Issues in Support of FGM/C}

From an interview with a TBA, it was revealed that FGM/C is performed at home for fear of the law and privacy that the home provides. Parents play a significant role in deciding whether their daughter should go through FGM/C or not. The discussions revealed that girls who do not go through the cut are regarded as

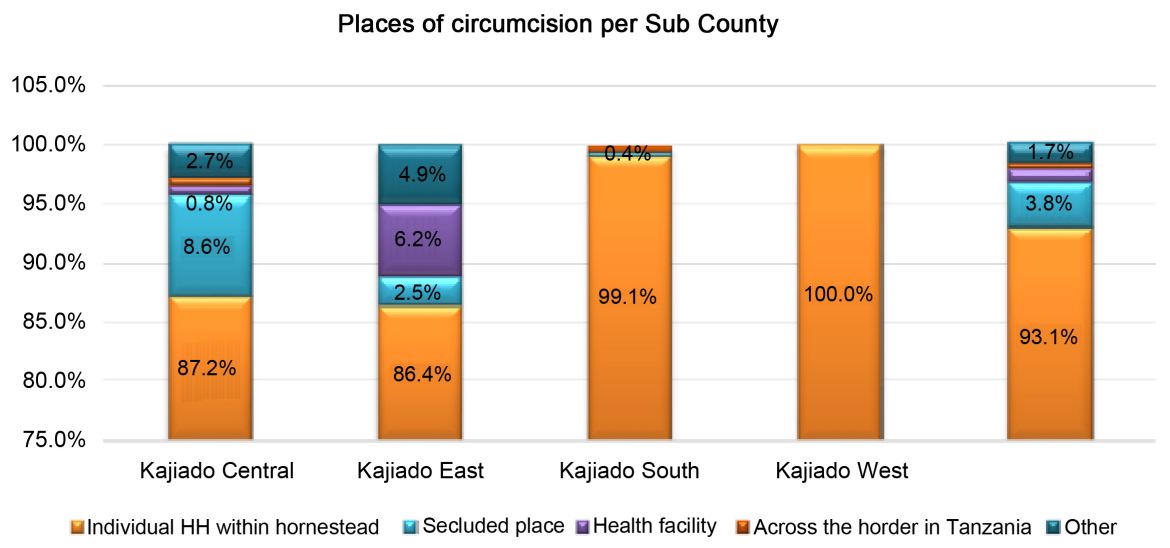

Figure 2. Places of circumcision per sub-county. 
children; they are disrespected and are not regarded as grown-ups; they are shunned by boys, ridiculed by peers and fear forceful circumcision during delivery. The community argues that they perform FGM/C for the purposes of a girl's social inclusion in the community; they are cut so that they can become wives, they are cut so that they can feel respected in the community. To fit within the circles of their peers, they will give in to the pressure of going through the cut to avoid ridicule. Culturally, it is a rite of passage to womanhood.

The community bestows a lot of respect from those who have undergone through the cut and it also comes with some status and cultural value. Women circumcision is indirectly linked to leadership i.e. the Maasai communities attach circumcision to capacity to lead. Additionally, they reported that girls would undergo FGM/C so as to make their parents, especially the father, proud and elevate him to another level amongst his age mates. It is also believed that the ceremony will appease the forefathers and ancestors.

\subsection{Cultural Justifications}

The Maasai community culture believes that an uncircumcised girl will not get suitors. Therefore, the culture dictates that a girl must be cut so that she can be married. It is a license for marriage. Girls are married off even before they attain the appropriate age for starting a family. It is also believed that cutting off the clitoris reduces sexual arousal especially at the adolescent stage when the urge for sex is high and at times not preventable. One of the discussants in Kajiado West said:

"...hawa wato to huwa na moto mingi sana..." (...These children are highly active sexually...).

Another driver for girls to give in to the pressure for FGM/C is that whenever they are pregnant, one who is uncircumcised cannot be helped to deliver as she is regarded as a coward. "No TBA will touch the blood of uncircumcised girls", one discussant said. It is believed that their blood is poisonous and can cause bad omen to whoever comes in contact with it. Additionally, it is believed that girls will go through the cut to avoid conflict and natural phenomena; for instance, drought and outbreaks of diseases that kill many people. Another discussant added that the community believes that miscarriages and increase in caesarian sections are also related to not being circumcised.

Culture and beliefs that uncircumcised girls are unhygienic are also some of the reasons given in support of FGM/C. It is believed that by circumcising a girl, you make her to be more hygienic. The term "Namuratuni" refers to the cleansing activity that the girl has to undergo. It does not have any link with physical hygiene and is usually a practice aimed at justifying that girls must be circumcised to remove a "worm" which to them (community) makes the girl unhygienic.

Girls who do not undergo the cut will always have pressure from the community, peers and from self in order to fit in their social circles. They are usually discriminated against and called names. They are referred to as "Entaapai or en- 
titobotor". This means that they cannot give their opinions, get a suitor nor get married and thus no bride price to the father.

\subsection{Economic Factors}

In the Maasai community, the girl child is highly valued, not because she will be educated to gain her position in the community, but because she will fetch some cows and goats as bride price to the father. The greatest beneficiaries from the practice are the men or the fathers of daughters. The men receive dowries from the suitors. It comes with some cultural incentives, a small gift to the girl's mother known as "the anointment" (esiret). The TBAs who perform FGM/C get paid in cash and kind and thus their hardline press on the need for all girls to undergo the cut. A discussant from an FGD with a group of a water point committee in Kilonito said:

"... The circumciser also gets incentives that include the tail of the sheep to make her hands soft and capable to circumcise many girls..."

\subsection{Knowledge and Perceptions of Community Members on FGM/C}

During female genital mutilation/cutting, excessive bleeding and pain are seen as signs of courage. The woman/girl is not supposed to cry and excessive bleeding is considered as "cleansing" of the girl and "getting rid of the childhood" in her. Since uncircumcised girls have not undergone the "cleansing" they are considered unclean "endapai" and TBAs cannot touch their blood during delivery because it is considered a bad omen. An interview with a TBA in Kajiado Central revealed that type II circumcision is practiced, where the clitoris and flesh are completely removed. This could help explain the excessive bleeding and pain associated with FGM/C.

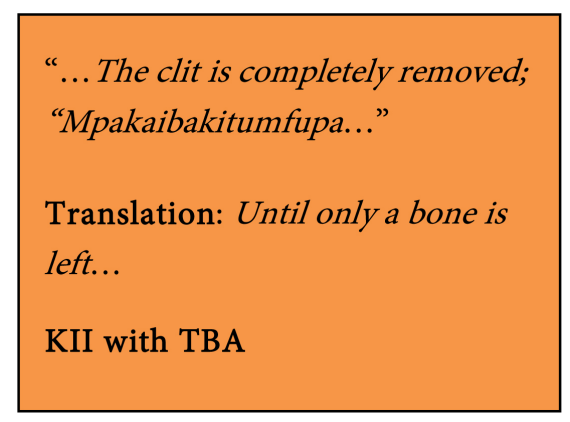

Furthermore, an FGD with women in Kajiado West revealed that if the cut was not properly done then it would be redone, and if a girl would try to resist because of pain, she is held still by other women and in some extreme cases the feet are tied apart. If the girl is too strong for the women, men would be requested to assist immobilize the girl whereby ropes would be tied to her feet and men would hold them from a distance. One discussant suggested that male involvement and dialogue with the key decision makers at the household level and 
at the community level is paramount including educating communities on effects of FGM for informed decisions.

\section{Discussion}

Although there are many interventions by Amref Health Africa and other partners, and support from the already existing anti-FGM/C laws that are aimed to end the practice, this study has shown that the practice is still rife in Kajiado County. We found that for them to evade arrest and prosecution, the TBAs carry out the cut in seclusion, mostly in the girl's homesteads to conceal the activity. Discussions with the circumcisers have also revealed that they have turned to cut younger age sets of girls even as young as 10 yrs. The high prevalence is also supported by the revelation that girls are usually cut to get suitors, as a source of wealth and respect for the girl's father amongst his peers, as well as to control promiscuity. Other push factors include cultural positions on birth and hygiene of girls that justify why they should undergo the cut.

Various research studies have primarily focused on establishing the prevalence rates between different countries, ethnic and age cohorts. In Kenya, no specific research study was found to have been done to establish the prevalence of FGM/C in Kajiado County, rather blanket prevalence among the Maasai pastoral communities. This study focused on finding out why the prevalence still remains high in Kajiado County despite campaigns and awareness creation fora, advocacy on enactment and enforcement of the existing legislation against FGM/C. The Alternative Rite of Passage (ARP) is one model offered to girls to graduate to womanhood without the cut. It is a socially acceptable means that takes girls through all the traditional and cultural processes including blessings by the traditional elders, coupled with training on their sexuality, reproductive health, and hygiene. This study found that there still push factors that will encourage the practice and these factors vary from the three reasons outlined above, social, cultural and economic, from the girls, parents and the community at large. Consequently, the alternatives are not adequately offered to all the parties involved for instance when a father to the girl fails to get respect from his peers. This revelation could, therefore, explain why even after laws are put in place to curb the vice, continuous sensitization and awareness campaigns, girls are still cut to satisfy their social acceptance in full knowledge of the dangers of the practice.

Our study had some limitations. There were respondents who chose not to take part in the survey. There is a possibility that those either the circumcised or non-circumcised women would have been over-represented, relative to the group that responded, in those who did not participate. This would lead to a biased estimate of the prevalence of FGM/C. However, given the high response rate $(95 \%)$ in our study the risk of such bias would have little impact on our prevalence estimates. We emphasized on the voluntary participation in the survey and no monetary benefits were extended to those who participated. 


\section{Conclusion and Recommendations}

FGM/C is a deeply rooted belief among the Maasai community in Kajiado County. The practice has no documented medical benefit to girls and women. Continuous sensitization on the effects of the practice, the enactment of the anti-FGM/C laws still not have the practice eliminated among the Maasai in Kajiado County. As evidenced by the discussions, the practitioners have only changed the timing. Cutting amongst younger age sets has been witnessed as well as secretly performing the act in closed doors within their households to conceal from the eye of the public.

The community is positive about the Amref's Alternative Rite of Passage of graduating girls to womanhood without going through the cut. Previous interventions have focused on the girl and mostly reactive. It is paramount to ensure that for the community's leaders who are the custodians of power own up the process, there must be deliberate efforts to include them in candid deliberations on the negative effects of the practice.

The alternatives need to be provided for all the beneficiaries and the process must be inclusive. The interventions must ensure that the rites of passage are community led/community driven and must always be preceded by rigorous community sensitizations where community leaders take lead. Additionally, the process should consider reducing social support for the practice rather than fully abandoned by practitioners and gradually the communities will understand the benefits of not circumcise their girls, and eventually advocate for total abandonment. This can be achieved by also coupling the process with initiatives that address gender and rights of women and girls and that demonstrate that women are also important stakeholders in development, for instance, scholarships for girls and placement in vocational training programs. Consequently, focus needs to be put in the social placement of men who intrinsically are the recipients of wealth as dowry as well as the social respect among his peers. If this social disturbance is not addressed, then the prevalence will still remain high. Therefore, there needs to come up with focused integrated interventions as alternatives to those who abandon the practice.

\section{Conflicts of Interest}

The authors declare no conflicts of interest regarding the publication of this paper.

\section{References}

[1] Fisaha, K.G. (2016) Female Genital Mutilation: A Violation of Human Rights. Journal of Political Sciences and Public Affairs, 4, 2-7.

http://www.esciencecentral.org/journals/female-genital-mutilation-a-violation-of-h uman-rights-2332-0761-1000198.php?aid=74616

https://doi.org/10.4172/2332-0761.1000198

[2] United Nations Children's Fund (2016) Female Genital Mutilation/Cutting: A Global Concern: UNICEF's Data Work on FGM/C Support for Data Collection, 
Data Analysis, and Dissemination. UNICEF.

https://www.unicef.org/media/files/FGMC_2016_brochure_final_UNICEF_SPREA D.pdf

[3] Ann-Marie, W. (2013) Country Profile: FGM in Kenya.

[4] Changing Embodied Practices : A Cultural Psychological Analysis of Female Genital Cutting and Alternative Rites of Research Report for Amref Health Africa July 2017.

[5] Wasike, A. and Krinninger, T. (2016) The Challenge of Eradicating FGM among Kenya's Maasai. DW, Africa.

http://www.dw.com/en/the-challenge-of-eradicating-fgm-among-kenyas-maasai/a19029709

[6] Njogu, G.N. (2015) The Influence of Female Genital Mutilation on Girl Child Participation in Public Primary Schools in Wamba Ward Samburu East Sub-County, Samburu County, Kenya. The University of Nairobi, Nairobi, Kenya.

[7] Substituting the "Cut": A Look into Alternative Rites of Passage-Youth For Change: A Global Platform for Girls' Rights Youth Activism. 